\title{
Carsten Reinemann \\ Medialisierung ohne Ende? \\ Zum Stand der Debatte um Medieneinflüsse auf die Politik
}

\section{Einleitung}

Medialisierung ist en vogue. ${ }^{1}$ Schwerpunktprogramme, Forschergruppen und universitäre Zentren werden ins Leben gerufen, die sich mit dem mutmaßlich wachsenden Einfluss der Medien auf die Gesellschaft beschäftigen. In den Blick genommen werden dabei nicht nur Veränderungen in der Politik, sondern in nahezu allen gesellschaftlichen Teilbereichen der Wirtschaft, der Religion, dem Recht und dem Sport bis zur Wissenschaft. Die Annahme einer wachsenden Relevanz medialer bzw. massenmedialer Kommunikation findet dabei zunehmend nicht nur in der Kommunikations-, sondern auch in anderen Geistes- und Sozialwissenschaften ihren Widerhall. Allerdings sind die zahlreichen derzeit anlaufenden Forschungsvorhaben ein deutlicher Hinweis darauf, dass es in der Medialisierungsforschung noch viel zu tun gibt. Die Kritik am derzeitigen Forschungsstand ist dementsprechend einhellig und massiv. So zog die ehemalige Präsidentin der größten internationalen kommunikationswissenschaftlichen Fachgesellschaft (ICA) Sonja Livingstone in ihrem Beitrag »On the mediation of everything « das ernüchternde Fazit: »In short, establishing the degree, nature, and consequences of the mediatization of anything or everything - politics, education, family, religion, self, - is a task largely ahead of us. ${ }^{2}$

Vor allem im Hinblick auf die Politik, die im Weiteren im Mittelpunkt dieses Beitrags stehen soll, muss dieses Fazit überraschend. Denn der wachsende Einfluss der Medien und dessen vermeintliche Folgen werden hier schon seit mehr als zwei Jahrzehnten auch außerhalb des wissenschaftlichen Diskurses debattiert. ${ }^{3}$ Dennoch werden auch im Hinblick auf die Medialisierung der Politik sowohl der Stand der Theoriediskussion als auch die Menge und Überzeugungskraft der empirischen Nachweise sehr kritisch beurteilt. So bemängeln Pfetsch und Marcinkowski (2009) in ihrem kürzlich erschienen Vorwort $\mathrm{zu}$ einem PVS-Sonderheft, dass es im Hinblick auf die Medialisierung an einem »breit angelegten, überzeugenden Forschungsprogramm« fehle und die Situation im Hinblick

1 Der vorliegende Beitrag ist eine überarbeitete Fassung der Antrittsvorlesung, die der Autor am 12. November 2009 vor der Sozialwissenschaftlichen Fakultät der Ludwig-Maximilians-Universität München gehalten hat.

2 Sonja Livingstone, »On the mediation of everything: ICA presidential address 2008 « in: Journal of Communication, Jg. 59, H. 1, (2009), S. 7.

3 Z.B. Heinrich Oberreuter, »Mediatisierte Politik und politischer Wertewandel « in: Frank E. Böckelmann, (Hg.), Medienmacht und Politik, Berlin 1989, S. 38. 
auf die empirischen Nachweise »prekär « sei: »Insbesondere stehen empirische Studien aus, die zeigen, dass die Medialisierung (...) nicht die Ausnahme, sondern die Regel der Medienperformanz und ihrer Verschränkung mit der Politik ist. «4

$\mathrm{Zu}$ dieser sehr skeptischen Einschätzung des Forschungsstandes trägt sicherlich die Unübersichtlichkeit des Forschungsfeldes bei, in dem sehr unterschiedliche Begriffsdefinitionen und Konzeptualisierungen konkurrieren und in dem vor dem Hintergrund ganz verschiedener theoretischer Ansätze eine Vielzahl empirischer Indikatoren verwendet werden. Das wichtigste Anliegen des vorliegenden Beitrags ist es deshalb, die bisherige Forschung vor allem im Hinblick auf ihre Begrifflichkeiten zu systematisieren, um dadurch zu einer klareren Konzeption von Medialisierung zu kommen. Sie soll eine Systematisierung und Aufarbeitung bisheriger Studien erleichtern und die künftige empirische Forschung von Ursachen, Indikatoren und Folgen der Medialisierung der Politik vorbereiten. Die eigentlich notwendige, umfassende Aufarbeitung bisheriger empirischer Studien kann hier nicht geleistet werden.

Jedoch sei der Hinweis erlaubt, dass wir auch empirisch eigentlich sehr viel mehr über die Medialisierung der Politik wissen, als es die oben zitierten, sehr pessimistischen Stimmen vermuten lassen. Allerdings werden die empirischen Befunde der deutschen und internationalen Forschung bisher nur ungenügend rezipiert. Dies liegt vermutlich nicht zuletzt daran, dass viele Studien nicht unter dem Label der »Medialisierung der Politik « publiziert wurden, sondern unter ganz anderen Schlagwörtern wie dem des »Agendabuilding « oder dem des »CNN-Effekts«. Doch selbst Studien, die den Terminus im Titel verwenden, werden kaum beachtet. ${ }^{5}$ Wir werden uns einer entsprechenden Aufarbeitung der empirischen Untersuchungen jedoch an anderer Stelle widmen und bemühen uns stattdessen hier darum, den Medialisierungsbegriff klarer zu konturieren.

\section{Die Medialisierung der Politik als Prozess}

Der Terminus Medialisierung wird in verschiedenen Disziplinen unterschiedlich verwendet. Verwirrung entsteht auf einer grundsätzlichen Ebene schon dadurch, dass mit Medialisierung unterschiedliche Medien-Begriffe und zeitliche Perspektiven verbunden werden.

Im Hinblick auf den Medienbegriff stehen auf der einen Seite Autoren, die Medien als technische Kommunikationsmittel verstehen. Sie interessieren sich für alle Formen dieser Kommunikation, unabhängig davon, ob sie öffentlich ist oder redaktionell von Journalisten der Massenmedien produziert wird. Hierunter fällt dann beispielsweise auch die private interpersonale Kommunikation über Mobiltelefone oder Emails und die oftmals geistes- oder kulturwissenschaftlich orientierte Forschung richtet sich unter anderem auf

4 Barbara Pfetsch, / Frank Marcinkowski, »Problemlage der Mediendemokratie Befunde zur Medialisierung der Politik « in: ders. (Hg.), Politik in der Mediendemokratie, PVS Sonderheft 42, Wiesbaden 2009, S. 17.

5 Z.B. Daniel Pontzen, Nur Bild, BamS und Glotze? Medialisierung der Politik aus Sicht der Akteure, Berlin/Hamburg/Münster 2006. 
die Folgen der technischen Vermittlung von Kommunikation für Kultur, Alltag und Identität der Menschen. ${ }^{6}$ Auf der anderen Seite stehen Autoren, die Medien als Mittel der Massenkommunikation verstehen und sich dementsprechend auf Prozesse öffentlicher Kommunikation konzentrieren, in denen redaktionell von Journalisten produzierte Medieninhalte eine zentrale Rolle spielen. ${ }^{7}$ In der Diskussion um die Medialisierung der Politik wird der Begriff der Medien in aller Regel in diesem Sinne verwendet.

Im Hinblick auf die zeitliche Perspektive wird von Medialisierung in einem statischen und in einem dynamischen Sinn gesprochen. In seiner statischen Verwendung bezeichnet der Begriff schlicht die Verarbeitung und Präsentation von Ereignissen, Themen oder Personen in und durch Massenmedien. Mit dem Medialisierung wird also beispielsweise darauf verwiesen, dass Ereignisse durch die spezifischen Regeln medialer Selektion, Produktion und Präsentation in einer spezifischen Weise sozial konstruiert werden. Durch ihre Medialisierung wird Realität zu Medienrealität. In diesem Sinne wird der Begriff oftmals im außereuropäischen, vor allem im US-Kontext verwendet. Altheide \& Snow ${ }^{8}$ etwa sprechen von »mediation «, Bennett $\&$ Entman ${ }^{9}$ sowie Nimmo \& Combs ${ }^{10}$ von » mediated politics «, Louw ${ }^{11}$ von » media-ization «. In seiner dynamischen Verwendung bezeichnet Medialisierung dagegen einen Prozess sozialen Wandels, in dessen Verlauf sich der Einfluss »der Medien « auf Akteure, Institutionen, deren Handeln und Interaktionen vergrößert bzw. größer geworden ist als derjenige anderer, nicht-medialer Akteure oder Institutionen. Oftmals ist hier von einer Anpassung an die »Logik der Medien « die Rede. Sie führe dazu, dass sich die Kriterien und Rationalitäten verändern, nach denen Akteure handeln und entscheiden. In diesem Sinne wird Medialisierung ${ }^{12}$ oder - meist synonym - Mediatisierung bzw. mediatization ${ }^{13}$ vor allem im europäischen Kontext verwendet (Abbildung 1).

6 Z.B. Friedrich Krotz, Mediatisierung. Fallstudien zum Wandel von Kommunikation, Wiesbaden 2007.

7 David L. Altheide, / Robert P. Snow, »Toward a theory of mediation « in: James A. Anderson, (Hg.), Communication Yearbook. Newbury Park/Beverly Hills/London/New Delhi 1988, Bd. 11, S. 194-223; und

Patrick Donges, »Medialisierung der Politik - Vorschlag einer Differenzierung « in: Patrick Rössler, / Friedrich Krotz, (Hg.), Mythen der Mediengesellschaft - The media society and its myths, Konstanz 2005, S. 321-339.

8 David L. Altheide, / Robert P. Snow, Media logic, Beverly Hills 1979.

9 W. Lance Bennett, / Robert M. Entman, Mediated politics: Communication in the future of democracy, Cambridge 2001.

10 Dan Nimmo, / James E. Combs, Mediated political realities, 2. Auflage, New York/London 1990.

11 Eric Louw, The media and the political process, London/Thousand Oaks/New Delhi 2005.

12 Z.B. Winfried Schulz, Politische Kommunikation: Theoretische Ansätze und Ergebnisse empirischer Forschung, Wiesbaden 2008; und Michael Meyen, »Medialisierung « in: Medien E Kommunikationswissenschaft, Jg. 57, H. 1 (2009), S. 23-38.

13 Z.B. Ulrich Sarcinelli, »Mediatisierung « in: Otfried Jarren, / Ulrich Sarcinelli, / Ulrich Saxer, (Hg.), Politische Kommunikation in der demokratischen Gesellschaft, Opladen 1998, S. 678-679; Gianpetro Mazzoleni, / Winfried Schulz, » >Mediatization< of politics: A challenge for democracy? in: Political Communication, Jg. 16, H. 3 (1999), S. 247-261; Gerhard Vowe, »Mediatisierung der Politik? Ein theoretischer Ansatz auf dem Prüfstand « in: Publizistik, Jg. 
Abbildung 1: Verwendungsweisen der Begriffe Medialisierung/Mediatisierung

\begin{tabular}{|c|c|c|c|}
\hline & & Medi & nbegriff \\
\hline & & $\begin{array}{l}\text { Mittel technisch } \\
\text { vermittelter Kom- } \\
\text { munikation } \\
\text { (privat oder offent- } \\
\text { lich) }\end{array}$ & $\begin{array}{l}\text { Mittel der } \\
\text { Massenkommunika- } \\
\text { tion (öffentlich) }\end{array}$ \\
\hline Zeitliche Perspektive & $\begin{array}{l}\text { statisch } \\
\text { (Ist-Zustand) }\end{array}$ & z.B. Krotz (2007) & $\begin{array}{l}\text { z.B. Altheide \& Snow } \\
\text { 1987; Bennet \& Ent- } \\
\text { man (2001); Louw } \\
(2005)\end{array}$ \\
\hline & $\begin{array}{l}\text { dynamisch } \\
\text { (Wandel) }\end{array}$ & z.B. Krotz (2007) & $\begin{array}{l}\text { z.B. Sarcinelli 1998; } \\
\text { Vowe 2006; Kepplin- } \\
\text { ger 2008; Schulz } 2008\end{array}$ \\
\hline
\end{tabular}

Eigene Darstellung.

Wir werden uns im Folgenden auf Medialisierung als dynamischen Prozess konzentrieren, in dem öffentliche Kommunikation in Massenmedien eine zentrale Rolle spielt. Es stellt sich dann in einem nächsten Schritt die Frage, wer oder was sich denn » medialisiert «, wenn von einer Medialisierung der Politik die Rede ist. Sarcinelli hat in diesem Zusammenhang eine recht weitreichende Verwendungsweise des Begriffs vorgeschlagen. Demnach bezeichnet Medialisierung dreierlei, nämlich die wachsende Verschmelzung von Medienwirklichkeit und politischer wie sozialer Wirklichkeit, die zunehmende Wahrnehmung von Politik im Wege medienvermittelter Erfahrung sowie die Ausrichtung politischen Handels und Verhaltens an den Gesetzmäßigkeiten der Medien. ${ }^{14}$

In Erweiterung bzw. Präzisierung dieser Definition halten wir es für sinnvoll, die Medialisierung der Politik an den drei Akteursgruppen festzumachen, die in modernen Massendemokratien das Dreieck politischer Kommunikation und Meinungsbildung darstellen. Als Objekte der Medialisierung können demnach Bürger, Medien und politische Akteure verstanden werden. Dem entsprechend lassen sich Medialisierungsphänomene auf der Mikro-, der Meso- und/oder der Makroebene konzeptualisieren und empirisch analysieren. Für alle drei Gruppen steigt im - angenommenen - Prozess der Medialisierung die Bedeutung von Massenmedien, massenmedialer Berichterstattung und/oder Medienlogik. Die drei genannten Elemente des Medienbegriffs sollen dabei auf institutionelle (Massenmedien), inhaltliche (Berichterstattung) bzw. die Handlungsrationalität

51, H. 4 (2006), S. 433-436; Stig Hjarvard, »The mediatization of society. A theory of the media as agents of social and cultural change « in: Nordicom Review, Jg. 29, H. 2 (2008), S. 105-134; Jesper Strömbäck, »Four phases of mediatization: An analysis of the mediatization of politics « in: The Harvard International Journal of Press/Politics, Jg. 13, H. 3 (2008), S. 228-246; und Hans Mathias Kepplinger, »Was unterscheidet die Mediatisierungsforschung von der Medienwirkungsforschung? « in: Publizistik, Jg. 53, H. 3 (2008), S. 326-338.

14 Vgl. Sarcinelli, Mediatisierung, aaO. (FN 13), S. 678 f.

ZfP 57. Jg. 3/2010 
betreffende Aspekte (Medienlogik) verweisen. Medialisierung der Politik kann also verstanden werden:

(1) als steigende Bedeutung von Medien, massenmedialer Berichterstattung und/oder Medienlogik für die Wahrnehmung und Beurteilung von Politik durch die Bürger sowie eine daraus vermutlich resultierende wachsende Bedeutung von Medien für die politischen Entscheidungen der Bürger, etwa für politische Partizipation, Wahlteilnahme oder die Richtung von Wahlentscheidungen. ${ }^{15}$

(2) als steigende Bedeutung von Medien, massenmedialer Berichterstattung und/oder Medienlogik für Selektions- und Präsentationsentscheidungen im Rahmen der politischen Berichterstattung der Massenmedien selbst. Damit ist beispielsweise gemeint, dass politische Überzeugungen oder Gemeinwohlorientierung zugunsten solcher Entscheidungskriterien in den Hintergrund gedrängt werden, die letztlich ökonomischen Erfolg sichern sollen, also die zunehmende Orientierung an Nachrichtenwerten wie Aktualität, Konflikt und Negativismus oder auch die Selbstreferentialisierung von Berichterstattung. ${ }^{16}$

(3) als steigende Bedeutung von Medien, massenmedialer Berichterstattung und/oder Medienlogik für die Wahrnehmungen und das Handeln politischer Akteure, wobei hier politische Akteure im engeren Sinn gemeint sind, also nicht die Bürger in ihren politischen Rollen, sondern vornehmlich Regierungen, Parteien, Interessenverbände und ihre Repräsentanten, aber durchaus auch Verwaltungen, Ministerien etc.

Auf Basis der bisherigen Überlegungen kann man die Medialisierung der Politik also wie folgt definieren:

Mit »Medialisierung der Politik ist ein Prozess sozialen Wandels gemeint, in dessen Verlauf die Bedeutung von Massenmedien, massenmedialer Berichterstattung und/ oder massenmedialer Logik für die politisch relevanten Wabrnehmungen und Handlungen von Bürgern, Medien und/oder politischen Akteuren zunimmt.

Wir werden uns im Folgenden vor allem mit der Medialisierung der Wahrnehmung und des Handelns politischer Akteure im engeren Sinne beschäftigen. Diesen Prozess wollen wir auch als Medialisierung politischer Akteure bezeichnen. Die Seite der Medien und Rezipienten werden wir nur in dem Maße berücksichtigen, in dem sie für die Veränderungen auf Seiten der Politik unmittelbar relevant ist. Will man nun die Medialisierung politischer Akteure als Prozess beschreiben und erklären, dann stellen sich vor allem drei Fragen: 1. Was sind die Ursachen der Medialisierung? 2. Was sind die Indikatoren der

15 Z.B. Sarcinelli, Mediatisierung, aaO. (FN 13); und Winfried Schulz, / Reimar Zeh, / Oliver Quiring, "Voters in a changing media environment: A data-based retrospective on consequences of media change in Germany « in: European Journal of Communication, Jg. 20, H. 1 (2005), S. 55-88.

16 Vgl. Kurt Imhof, »Mediengesellschaft und Medialisierung « in: Medien und Kommunikationswissenschaft, Jg. 54, H. 2 (2006), S. 191-215; Otfried Jarren, / Werner A. Meier, (Hg.), Ökonomisierung der Medienindustrie: Ursachen, Formen und Folgen. Themenheft Medien \& Kommunikationswissenschaft 49/2, Baden-Baden 2001; und Carsten Reinemann, / Jana Huismann, »Beziehen sich Medien immer mehr auf Medien? Dimensionen, Belege, Erklärungen « in: Publizistik, Jg. 52, H. 4 (2007), S. 465-484. 
Medialisierung, d.h. woran erkennt man eine veränderte Bedeutung von Medien, Medienberichterstattung oder Medienlogik? 3. Was sind die Folgen der Medialisierung?

Wie Donges deutlich macht, sind Ursachen, Indikatoren und Folgen oftmals Bestandteil der Definitionen von Medialisierung. ${ }^{17}$ Dies führt zum Teil dazu, dass Ursachen und Folgen als bekannt vorausgesetzt und nicht mehr hinterfragt werden. Genau dies aber wollen wir im Folgenden tun. Dabei wird deutlich werden, dass die Medialisierungsforschung vor allem im Hinblick auf die Konzeptualisierung und empirische Untersuchung von Ursachen und Folgen der Medialisierung noch am Anfang steht. Es dominieren vielmehr Thesen und Vermutungen auf recht dünner empirischer Basis.

\section{Ursachen der Medialisierung politischer Akteure}

Im Hinblick auf die Ursachen eines verstärkten Medieneinflusses stellen sich vor allem zwei Fragen, die bislang nicht hinreichend differenziert und geklärt worden sich. Zum einen, welchen Beitrag zur Medialisierung die Medien selbst und welchen Beitrag andere Prozesse gesellschaftlichen Wandels geleistet haben. Zum zweiten, wenn man einen kausalen Einfluss des Medienwandels annimmt, welche Veränderungen der Medien, der Medienberichterstattung oder der Medienlogik es denn nun genau sein sollen, die einen stärkeren Medieneinfluss bedingen. Die Unklarheit über die Ursachen der Medialisierung wird dabei auch darin deutlich, dass sich in der Literatur ganz unterschiedliche Einschätzungen dazu finden, $\mathrm{ab}$ wann überhaupt von einer Medialisierung der Politik gesprochen werden kann. Während einige Autoren diese am Aufkommen des Fernsehens ${ }^{18}$ oder später bei der Einführung des dualen Rundfunks ansetzen, ${ }^{19}$ sehen andere bereits mit dem Aufkommen der Massenpresse erste Medialisierungsschübe. ${ }^{20}$

Die Kommunikationswissenschaft neigt in der Medialisierungsdiskussion dazu, Veränderungen der Medien als ursächlich für Medialisierungsprozesse zu betrachten. ${ }^{21}$ Manche Autoren bezeichnen nur das als Medialisierung, was sich tatsächlich durch mediale Veränderungen erklären lässt. Hat man allerdings die veränderte Bedeutung von Medien für politische Akteure als abhängige Variable im Blick - also etwa einen Wandel ihrer Handlungsrationalität oder eine stärkere Beachtung von Medienberichterstattung -, dann könnte eine solche Medialisierung ihres Handelns natürlich auch völlig ohne

17 Vgl. Donges, Medialisierung der Politik - Vorschlag einer Differenzierung, aaO. (FN7), S. 324; Michael Meyen, »Medialisierung « in: Medien E Kommunikationswissenschaft, Jg. 57, H. 1 (2009), S. 23-38; Winfried Schulz, Politische Kommunikation: Theoretische Ansätze und Ergebnisse empirischer Forschung, Wiesbaden 2008; und Hjarvard, The mediatization of society. A theory of the media as agents of social and cultural change, aaO. (FN 13), S. 105-134.

18 Winfried Schulz, Reconstructing mediatization as an analytical concept « in: European Journal of Communication, Jg. 19, H. 1 (2004), S. 87-101.

19 Imhof, Mediengesellschaft und Medialisierung, aaO. (FN16), S. 191-215.

20 Frank Bösch, / Norbert Frei, »Die Ambivalenz der Medialisierung. Eine Einführung « in: ders. (Hg.), Medialisierung und Demokratie im 20. Jahrbundert, Göttingen 2006, S. 7-23.

21 Vgl. Meyen, Medialisierung, aaO. (FN 17), S. 23-38; und Andrea Schrott, »Dimensions: CatchAll Label OR Technical Term « in: Knut Lundby, (Hg.), Mediatization. Concept, Changes, Consequences, New York 2009, S. 41-61. 
mediale Veränderungen zustande gekommen sein. So könnten sich politische Akteure in ihrem Handeln an journalistische Kriterien der Selektion und Präsentation von Nachrichten allein schon deshalb angepasst haben, weil sich infolge des sozialen Wandels die traditionellen Parteiloyalitäten auflösen und es die Politik mit einer wachsenden Zahl von Wechselwählern und Spätentscheiden zu tun hat, von denen man annimmt, dass sie für Wirkungen massenmedial vermittelter politischer Kommunikation besonders anfällig sind. ${ }^{22}$

Es ist also nach wie vor eine offene empirische Frage, ob der Medienwandel die primäre Ursache der Medialisierung ist, ob dieser eine unter mehreren Ursachen darstellt oder ob medialer und politischer Wandel nur parallel verlaufen. Ja, vermutlich gibt es einen Beitrag medialer Veränderungen zur Medialisierung der Politik. Wahrscheinlich ist aber auch, dass Entwicklungen wie beispielsweise die Auflösung traditioneller Milieus und die damit einhergehende Abnahme von Parteibindungen in der Bevölkerung sowie die zunehmende Volatilität politischer Stimmungen wohl immensen Einfluss auf die Medialisierung des Handelns politischer Akteure gehabt haben. Um es auf den Punkt zu bringen: Angesichts abnehmender politischer Loyalitäten hätte sich die Politik wohl auch dann stärker an die Medien angepasst, wenn sich die Medien selbst gar nicht verändert hätten. Realistischerweise ist deshalb davon auszugehen, dass sich medialer, gesellschaftlicher und politischer Wandel wechselseitig bedingen und in ihrer Interaktion für Medialisierungsprozesse verantwortlich sind. ${ }^{23}$ Eine theoretische Rekonstruktion dieser gegenseitigen Einflussprozesse steht allerdings noch aus.

Unbefriedigend ist bislang auch die Frage beantwortet, welche Aspekte medialen Wandels in welche Weise mit welchen Medialisierungsphänomenen in Beziehung stehen sollen. In der Literatur wird Medialisierung häufig relativ abstrakt auf einen »Strukturwandel des Mediensystems «, »den Medienwandel « oder auch schlicht auf »gesellschaftlichen Wandel« zurückgeführt. ${ }^{24}$ Zuweilen wird Medialisierung aber auch als Metaprozess charakterisiert, der sich eigentlich gar nicht recht erklären ließe. Für die sozialwissenschaftliche Analyse eines gesellschaftlichen Phänomens wäre der Begriff damit aber weitgehend unbrauchbar. ${ }^{25} \mathrm{Im}$ Hinblick auf die Medialisierung politischer Akteure sollte in jedem Fall intensiver diskutiert werden, welche Aspekte des Medienwandels denn nun genau zu welchen Veränderungen auf Seiten der politischen Akteure geführt haben sollen?

Ist die Ursache der Medialisierung politischer Akteure die allgemeine Medienexpansion, die sich in der enormen Ausweitung und Differenzierung von Medienangeboten

22 Carsten Reinemann, / Marcus Maurer, / Olaf Jandura, / Thomas Zerback, »Die Spätentscheider. Wer sind sie und wie entscheiden sie sich?« in Heinrich Oberreuter, (Hg.), Der Bundestagswablkampf 2009 (Arbeitstitel), München 2010 (im Vorbereitung).

23 Z.B. Christina Holtz-Bacha, »Professionalisation of politics in Germany « in: Ralph Negrine, / Paolo Mancini, / Christina Holtz-Bacha, / Stylianos Papathanassopoulos, (Hg.), The professionalisation of political communication, Chicago 2007, S. 63-79; und Schulz, Politische Kommunikation: Theoretische Ansätze und Ergebnisse empirischer Forschung, aaO. (FN 17).

24 Schrott, Dimensions: Catch-All Label OR Technical Term, aaO. (FN 21), S. 41-61.

25 Vgl. z.B. Krotz, Mediatisierung. Fallstudien zum Wandel von Kommunikation, aaO (FN 6). 
zeigt? Sind es bestimmte technische Innovationen (z.B. Digitalisierung)? Ist es der Siegeszug des Fernsehens oder neuerdings der Online-Medien? Ist es die Deregulierung im Rundfunkbereich, die zur Entstehung des dualen Rundfunksystems geführt hat? Sind es wachsende Konkurrenz, Ökonomisierung und Publikumsorientierung im Journalismus? Sind es die infolgedessen sich verändernden inhaltlichen Strukturen der Medienangebote, die mit Schlagwörtern wie Unterhaltungsorientierung, Visualisierung, Personalisierung, Negativismus oder Konfliktorientierung umschrieben werden? Sind es die veränderten journalistischen Arbeitsweisen, die sich etwa in einer wachsenden Beschleunigung und Selbstreferentialisierung des Journalismus zeigen? Oder ist es gar nicht primär der Medienwandel selbst, sondern vor allem die Veränderung der Bedeutung der Medien als Quelle politischer Information der Bürger? Oder ist es die Veränderung der Nutzungsmuster, die zunehmende Zersplitterung des Publikums oder die durch veränderte Berichterstattungsmuster sich verändernden Kriterien der Bürger bei der Beurteilung von Politik? Und vor allem: In welcher Art und Weise wurden diese Veränderungen von den politischen Akteuren wahrgenommen und wie haben sie zu welchen konkreten Veränderungen von Handlungsweisen oder organisatorischen Strukturen geführt?

Angesichts der unklaren Ursachen einer möglichen Medialisierung ist es sinnvoll, diese möglichen Ursachen nicht zum Bestandteil einer Definition von Medialisierung zu machen. Vielmehr halten wir es für sinnvoll, in Erweiterung der oben vorgeschlagenen Definition von einer Kausalkette auszugehen, die die Ursachen der Medialisierung zunächst offen lässt und stattdessen die Wahrnehmungen der politischen Akteure in den Blick nimmt. Eine solche Kausalkette regt einerseits dazu an, ein breites Spektrum möglicher Faktoren als Ursachen der Medialisierung in den Blick zu nehmen und richtet andererseits den Blick auf das mögliche Missverhältnis von wahrgenommenen und realen Medieneinflüssen. In diesem Sinne kann man davon ausgehen, dass die Ursachen der Medialisierung sich folgendermaßen beschreiben lassen:

Die Ursache der Medialisierung politischer Akteure bestebt in der Annabme der Akteure, dass die Wirkungschancen von Medien auf sie selbst, auf Wäbler, auf politische Freunde oder Gegner größer geworden sind. Sie neigen daher eher dazu, mögliche Wirkungen von Medienberichterstattung zu antizipieren und sie richten ibr Handeln bei der Darstellung und/oder Herstellung von Politik deshalb verstärkt an massenmedialer Berichterstattung und/oder Medienlogik aus.

Folgt man dieser Sichtweise, dann ist der Ausgangspunkt der Medialisierung nicht notwendiger ein tatsächlicher Wandel von Medien oder Medieneinflüssen, sondern zunächst einmal die veränderte Wabrnehmung von Medieneinflüssen. Medialisierung ergibt sich also zunächst daraus, dass politische Akteure Medien als wirkungsvoll bzw. wirkungsvoller wahrnehmen ${ }^{26}$ und diese Vorstellungen handlungsleitend werden. Dementsprechend sind die bei politischen Akteuren auftretenden Verzerrungen in der Wahrnehmung von Medienberichterstattung (z.B. Hostile Media Phenomenon), in den Annahmen über Medieneffekte (z.B. Third-Person-Effekt) sowie dies daraus resultierenden 
Auswirkungen auf politisches Handeln von zentraler Bedeutung für die Medialisierungsforschung. Nicht umsonst werden diese psychologischen Konzepte zunehmend zum Gegenstand kommunikationswissenschaftlicher Forschung und Theoriebildung (z.B. reziproke Effekte; ${ }^{27}$ Theory of presumed media influence). ${ }^{28}$

\section{Indikatoren der Medialisierung politischer Akteure}

Definiert man die Medialisierung politischer Akteure in der skizzierten Weise, dann stellt sich in einem nächsten Schritt die Frage, wie politische Akteure auf die wahrgenommene Bedeutungssteigerung der Medien konkret reagieren? Was also sind die Indikatoren der Medialisierung? Sucht man nach solchen Indikatoren, dann wird man nicht nur in der Literatur zur Medialisierung, sondern auch in vielen Beiträgen fündig, die sich generell mit dem Verhältnis von Politik und Medien auseinandersetzen.

Auf einer relativ abstrakten Ebene wird oftmals von einer Anpassung der Politik an die Medienlogik ${ }^{29}$ oder von einer Ausrichtung der Politik an der Medienlogik ${ }^{30}$ gesprochen. Andere Autoren bevorzugen den Begriff der Reaktion, um deutlich zu machen, dass politische Akteure und Organisationen verschiedene Möglichkeiten haben, mit den Anforderungen der Mediengesellschaft umzugehen. ${ }^{31}$ Eher abstrakte Indikatoren werden auch in verschiedenen Phasenmodellen genannt, die die Entwicklung der Medialisierung der Politik beschreiben wollen. ${ }^{32}$ Betrachtet man konkretere Indikatoren, dann finden sich darunter sowohl solche, die sich primär auf die Darstellung von Politik beziehen, als auch solche, die sich auf die Herstellung von Politik beziehen, also auf die inhaltliche Substanz und damit den Kern des politischen Prozesses.

$\mathrm{Zu}$ den Indikatoren für eine Medialisierung der Darstellung von Politik gehören unter anderem (a) eine generell größere »Offenheit « für Medien und Journalisten; (b) die $\mathrm{Zu}$ nahme von Medienaktivitäten bzw. des dafür aufgewandten zeitlichen oder finanziellen Budgets (z.B. für Kontakte zu Journalisten, Medienauftritte, Medienmonitoring, Pressearbeit, Inszenierung von Kampagnen); (c) die Professionalisierung von Medienaktivitäten, die sich in individuellen Aktivitäten wie Medientrainings, aber auch in strukturellen Änderungen wie der Einrichtung von Pressestellen oder Rapid-Response Units, deren organisatorischer Verortung (etwa als Stabsstelle), der Personalrekrutierung und der Hinzuziehung externer Experten niederschlagen kann; (d) die zunehmend mediengerechte öffentliche Kommunikation, die sich beispielsweise in einer Vereinfachung, Ver-

27 Hans Mathias Kepplinger, »Politiker als Protagonisten der Medien« in: Zeitschrift für Politik, Jg. 54, S. S. 274-295.

28 Jonathan Cohen, / Yariv Tsfati, / Tamir Sheafer, »The influence of presumed media influence in politics: Do politicians' perceptions of media power matter? « in : Public Opinion Quarterly, Jg. 1 (2008), S. 1-14.

29 Schulz, Reconstructing mediatization as an analytical concept, aaO. (FN 18), S. 87-101.

30 Vgl. Sarcinelli, Mediatisierung, aaO. (FN 13), S. 678-679.

31 Patrick Donges, Medialisierung politischer Organisationen. Parteien in der Mediengesellschaft, Wiesbaden 2008, S. 218.

32 Z.B. Jesper Strömbäck, »Four phases of mediatization: An analysis of the mediatization of politics « in: The Harvard International Journal of Press/Politics, Jg. 13, H. 3 (2008), S. 228-246. 
kürzung, Zuspitzung etc. von Sachverhalten, Argumentationen und Entscheidungsprozessen zeigen kann.

Zur Systematisierung der Indikatoren, die sich unmittelbar auf die Herstellung von Politik, also den Kern politischer Personal- und Policy-Entscheidungen beziehen, kann man typische Modelle von Politik-Zyklen heranziehen. Dann kann sich eine Medialisierung politischer Akteure nicht »nur « in der (a) Problemdefinition und (b) der Themensetzung für die Politik, sondern auch in der (c) Formulierung und Auswahl von Policy-Alternativen sowie deren (d) Implementation und (e) Evaluation zeigen. Dass solche Einflüsse von Medien auf politische Akteure nicht nur in der Theorie, sondern auch in der Praxis prinzipiell bestehen, dies zeigen nicht nur diverse internationale Studien, die sich ganz unterschiedlicher Methoden und Designs bedienen und unterschiedliche Akteure, Politikebenen, Politikfelder und Ländern untersuchen. ${ }^{33}$ Auch für Deutschland gibt es deutliche Hinweise auf solche substanziellen Einflüsse. Sie stammen nicht nur aus Anekdoten, die sich immer wieder in der alläglichen Medienberichterstattung finden. Auch wissenschaftliche Studien sind hier zu nennen. So gab ein Drittel der Bundes- und Landtagsabgeordneten in einer Befragung aus dem Jahr 2005 an, die Medien hätten bei der Themensetzung für die Politik mehr Einfluss als diese selbst. Ein weiteres Drittel der Abgeordneten sah hier ein Gleichgewicht von Politik und Medien. Ein weiterer Befund: Fast die Hälfte der Befragten war der Ansicht, die Chancen eines komplexen und nicht medientauglichen Themas, überhaupt in den Gesetzgebungsprozess zu kommen, seien heute geringer als früher. ${ }^{34}$

Leider werden die unterschiedlichen Facetten von Medialisierung oftmals nicht hinreichend differenziert. Dann wird zuweilen von einer Medialisierung der Darstellung auf eine Medialisierung der Herstellung von Politik geschlossen oder umgekehrt eine Medialisierung ihrer Herstellung a priori ausgeschlossen..$^{35}$ Für die Frage der gesellschaftlichen Relevanz der Medialisierungsforschung, aber auch für normative Beurteilung einer Medialisierung des Handelns politischer Akteure, ist die Differenzierung zwischen der Form und dem Inhalt von Politik dabei jedoch von fundamentaler Bedeutung. Bezieht sich eine Medialisierung allein auf die im Zweifel professionellere Darstellung von Politik, dann sind die Folgen einer Medialisierung wohl bei weitem nicht so relevant oder auch problematisch, als wenn sich die Substanz der Politikherstellung den Gesetzmä-

33 Z.B. Matthew A. Baum, Soft news goes to war. Public opinion and American foreign policy in the new media age, Princeton 2003; Cohen et al., The influence of presumed media influence in politics: Do politicians' perceptions of media power matter? aaO. (FN 29), S. 1-14.; Bryan D. Jones, / Frank R. Baumgartner, The politics of attention: How government prioritizes problems, Chicago/London 2005; Martin Linsky, Impact: How the press affects federal policymaking, New York 1986; Stefaan Walgrave, / Peter van Aelst, »The contingency of the mass media's political agenda setting power. Toward a preliminary theory « in: Journal of Communication, Jg. 56, H. 1 (2006), S. 88-109; und Stefaan Walgrave, »Again, the almighty mass media? The media's political agenda-setting power according to politicians and journalists in Belgium« in: Political Communication, Jg. 25, H. 4 (2008), S. 445-459.

34 Vgl. Pontzen, Nur Bild, BamS und Glotze? Medialisierung der Politik aus Sicht der Akteure, aaO. (FN 5), S. 93.

35 Vgl. Karl-Rudolf Korte, / Manuel Fröhlich, Politik und Regieren in Deutschland: Strukturen, Paderborn 2006. S. 15. 
Bigkeiten medialen Erfolgs oder den Konjunkturen medialer Berichterstattung unterwerfen würde. Wir wollen deshalb in Abhängigkeit davon, ob die Medialisierung die Herstellung oder die Darstellung von Politik betrifft, von unterschiedlichen Graden der Medialisierung sprechen (Abbildung 2):

Die Medialisierung politischer Akteure kann sowobl die Darstellung als auch die Herstellung von Politik betreffen. Je eher sich die Medialisierung auch auf Handlungen bezieht, die der Herstellung von Politik zuzurechnen sind, desto böher ist der Grad der Medialisierung des politischen Handelns. Je eher sich die Medialisierung vor allem auf Handlungen bezieht, die vor allem der Darstellung von Politik zuzurechnen sind, desto niedriger ist der Grad der Medialisierung der Politik.

Abbildung 2: Indikatoren für die Medialisierung politischer Akteure

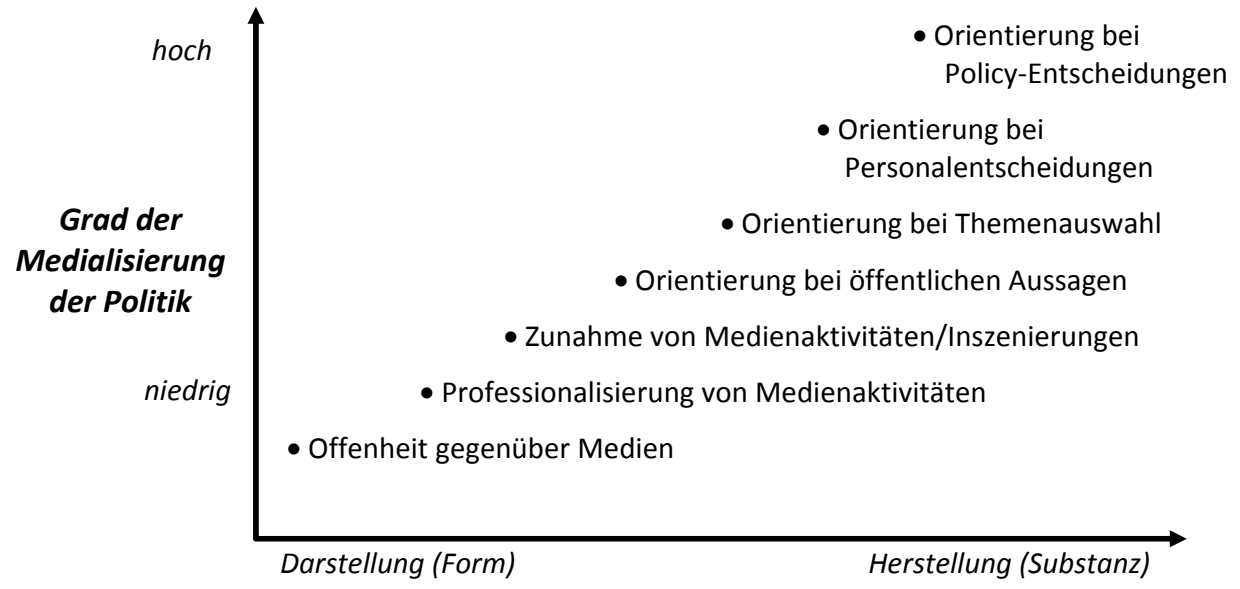

Dimension von Politik

Eigene Darstellung

\section{Folgen der Medialisierung politischer Akteure}

In der Diskussion um die Medialisierung politischer Akteure werden vor allem negative Folgen dieser Entwicklung befürchtet. Die möglichen Folgen beziehen sich auch auf die Rückwirkungen der Medialisierung politischen Handelns auf Medien und Rezipienten, insbesondere in der politikwissenschaftlichen Diskussion überwiegt jedoch eine Thematisierung von Medialisierungsfolgen für die Politik selbst. So ist nicht nur von einem Autonomie-, sondern auch von einem Machtverlust der Politik gegenüber den Medien die Rede. Allerdings finden sich durchaus auch Stimmen, die zwar eine Anpassung der Politik an die Medienlogik konstatieren, damit aber nicht unbedingt einen Machtverlust der Politik verbunden sehen wollen. Für diese Autoren ist die Antizipation von Medienlogik durch politische Akteure zuvorderst ein Mittel zum Machterhalt und eine Möglichkeit, die Chancen zur Instrumentalisierung von Medien zu erhöhen. Wer die Selek- 
tions- und Präsentationsregeln der Medien kennt und antizipiert, so die Argumentation, kann dieses Wissen durch geschicktes Kommunikationsmanagement zum eigenen Vorteil nutzen. Er verliert dadurch nicht etwa an Autonomie und an Macht über die Medien und deren Publikum, sondern bringt sich in eine bessere Position zur Erreichung seiner Ziele. ${ }^{36}$ Am überzeugendsten ist eine solche Argumentation dann, wenn sie sich auf die Darstellung von Politik bezieht. Geht es um die Substanz von Politik, wird Anpassung sehr viel eher mit Machtverlust einhergehen.

Dass die Medialisierung des Handelns politischer Akteure nicht unbedingt mit einem Machtverlust einhergehen muss, legen im übrigen auch parallel ablaufende Wandlungsprozesse in Medien und Journalismus nahe, die den Erfolg von Instrumentalisierungsversuchen wahrscheinlicher machen. So werden deutsche Journalisten angesichts des wachsenden ökonomischen Drucks und schwindender Ressourcen für die journalistische Arbeit (z.B. Recherche) zunehmend abhängig von ihren Quellen in Wirtschaft, Politik, Gesellschaft und anderen Medien. ${ }^{37}$ Nicht zwingend ist die Annahme eines Machtverlusts durch Medialisierung auch deshalb, weil ihr zumindest implizit eine linear-deterministische Vorstellung von sozialem Wandel zugrunde liegt. Sie lässt mögliche Gegenreaktionen von Akteuren unberücksichtigt. So kann die Antizipation von Medienlogik oder Medienberichterstattung auch dazu führen, dass politische Akteure Vorhaben, Überlegungen oder Handlungsalternativen nicht öffentlich kommunizieren. Dies wäre aus Sicht mancher realistischer Demokratietheorie vielleicht als notwendig und funktional anzusehen, aus Sicht anderer demokratietheoretischer Vorstellungen jedoch ein problematischer Verlust an politischer Transparenz. ${ }^{38}$ Schließlich bleibt in dem scheinbaren Nullsummenspiel der Einflussverschiebung von der Politik auf die Medien unberücksichtigt, dass die eigentlichen Einflusszuwächse in den letzten Jahren unter Umständen gerade bei jenen neuen Akteuren zu suchen sind, die die Governance-Forschung in den Blick nimmt und die an politischer Öffentlichkeit unter Umständen gar kein oder nur dann Interesse haben, wenn sie die Politik unter Druck setzen wollen (z.B. Unternehmen, Verbände, NGOs).

Zusätzlich zur eher recht abstrakten These einer generellen Machtverschiebung zu den Medien werden noch weitere negative Folgen der Medialisierung befürchtet. So findet sich die Annahme, dass Medienkompetenz anstelle von Sachkompetenz zum zentralen Erfolgsfaktor für Politiker würden; ${ }^{39}$ dass die Parteien sich zunehmend zentrali-

36 Z.B. Daria W. Dylla, »Der Einfluss politischer Akteure auf die Politikberichterstattung. Selbstmedialisierung der Politik? in: Thomas Jäger, / Henrike Viehrig, (Hg.), Die amerikanische Regierung gegen die Weltöffentlichkeit?: Theoretische und empirische Analysen der Public Diplomacy zum Irakkrieg, Wiesbaden 2008, S. 53-76.

37 Z.B. Siegfried Weischenberg, / Maja Malik, / Armin Scholl, Die Souffleure der Mediengesellschaft. Report über die Journalisten in Deutschland, Konstanz. 2006.

38 Dazu Philip Baugut, / Maria-Theresa Grundler, Politische (Nicht-)Öffentlichkeit in der Mediendemokratie. Eine Analyse der Beziehungen zwischen Politikern und Journalisten in Berlin. Baden-Baden 2009.

39 Z.B. Danilo Zolo, Die demokratische Fürstenherrschaft, Göttingen 1997, S. 200 f. 
sieren und von ihrem Spitzenpersonal abhängig machen würden; ${ }^{40}$ dass die Etablierung der Medien als neue Politikarenen zu einer Sinnentleerung der Parlamente beitrügen. ${ }^{41}$ Aber auch massive Auswirkungen auf die Strukturen des politischen Prozesses werden befürchtet. So ist von einer geringeren Thematisierungskompetenz der Politik und einer zunehmenden Hinwendung zu medien-adäquaten Themen die Rede. ${ }^{42}$ Befürchtungen betreffen außerdem die Frage, ob politische Akteure überhaupt noch den Versuch machen, politische Konzepte und Entscheidungen öffentlich zu erklären bzw. die Bürger von ihnen zu überzeugen. Und die Befürchtungen reichen auch in den Kern politischer Entscheidungen, wenn die These vertreten wird, symbolische Politik und Populismus könnten als Folge der Medialisierung um sich greifen und die Medialisierung führe zu einer Abnahme der Qualität politischer Entscheidungen. ${ }^{43}$

Allerdings gibt es bislang keinerlei Studien, die den Versuch machen, solche weit reichenden Folgen der Medialisierung auch empirisch nachzuweisen. Ebenso wenig werden mögliche positive Folgen einer Medialisierung der Politik diskutiert, etwa im Sinne einer Steigerung von kommunikativer Kompetenz auf Seiten der politischen Akteure oder im Sinne von gesteigerter Responsivität. Dies liegt vor allem daran, dass zuweilen übersehen wird, dass der Grund für eine möglicherweise gewachsene Medienorientierung in erster Linie nicht die Medien selbst, sondern deren Wirkungen auf andere politische Akteure oder die Bürger sind. Auch hier liegt noch ein großes Potential für künftige Forschung.

\section{Ausblick: Theoretische und empirische Herausforderungen}

Nachdem wir der Vielzahl von Diskussionsbeiträgen zur Medialisierung der Politik einen weiteren hinzugefügt haben, möchten wir in der gebotenen Kürze noch einen Ausblick auf die theoretischen und empirischen Herausforderungen werfen, vor denen die Medialisierungsforschung derzeit steht:

Aus theoretischer Sicht stecken in der Medialisierungsdiskussion eine Fülle von Annahmen: Über Wahrnehmungsprozesse von Akteuren; über die Antizipation von Medienwirkungen, die Einfluss auf die Darstellung oder Herstellung von Politik nehmen können; über grundsätzliche Veränderungen der Handlungsrationalität politischer Akteure; über Dynamiken sozialen Wandels und über mögliche Folgen der Medialisierung

40 Z.B. Ulrich von Alemann / Stefan Marschall, »Parteien in der Mediendemokratie - Medien in der Parteiendemokratie in: ders. (Hg.), Parteien in der Mediendemokratie, Wiesbaden 2002, S. $28 \mathrm{ff}$.

41 Z.B. Stefan Marschall, »Das Parlament in der Mediengesellschaft - Verschränkungen zwischen parlamentarischer und massenmedialer Arena « in: Politische Vierteljabresschrift, 42, 3 (2001), S. 407.

42 Z.B. Wolfgang Hüning, / Jens Tenscher, »Medienwirkungen von Parteistrategien. AgendaBuilding-Prozesse im nordrhein-westfälischen Landtagswahlkampf 2000 « in: Ulrich Sarcinelli, / Heribert Schatz, (Hg.), Mediendemokratie im Medienland?, Opladen 2002, S. 289-317; und Walgrave, / van Aelst, The contingency of the mass media's political agenda setting power. Toward a preliminary theory, aaO. (FN 35), S. 88-109.

43 Pfetsch / Marcinkowski, >Problemlage der Mediendemokratie . Theorien und Befunde zur Medialisierung der Politik, aaO. (FN4), S. 11-33. 
auf Mikro-, Meso- und Makroebene. Ein theoretischer Rahmen, innerhalb dessen sich Medialisierungsprozesse sinnvoll beschreiben und erklären lassen, muss idealerweise die Möglichkeit bieten, alle diese Annahmen zu konzeptualisieren und in empirische Forschung zu überführen. Dabei sollte er anschlussfähig sein an kommunikations- und politikwissenschaftliche Konzepte der Journalismus-, Rezeptions-, Medienwirkungs-, Wahl- und Einstellungs- sowie der Policy-Forschung. Die in der Literatur bislang bevorzugten handlungs- ${ }^{44}$ institutionen- ${ }^{45}$ und systemtheoretischen Ansätze ${ }^{46}$ erfüllen diese Kriterien unterschiedlich gut. Wir plädieren hier für die Anwendung eines akteursorientiterten Paradigmas wie dem des strukturell-individualistischen Ansatzes. ${ }^{47}$ Einer der Vorteile dieses Ansatzes ist, dass er ein ausdifferenziertes Konzept sozialen Wandels bereithält, das sich als Heuristik für die Rekonstruktion der mit der Medialisierungsannahme verbundenen Prozesse und Interaktionen eignet. Zudem ermöglicht er die Explizierung von Mikro-, Meso-, Makro-Links und erlaubt es, die Differenzen von Akteuren und Situationen zu konzeptualisieren. Diese Möglichkeit ist außerordentlich bedeutsam. Denn wenn es ein halbwegs konstantes Ergebnis der bisherigen empirischen Forschung zur Medialisierung politischer Akteure gibt, dann dies, dass es offensichtlich (1) erhebliche stabile Differenzen zwischen der Her- und Darstellung von Politik, zwischen Akteuren, Politikfeldern und Phasen des Politik-Zyklus gibt sowie (2) erhebliche situationale Differenzen bestehen, etwa in Abhängigkeit davon, ob man sich in Wahlkämpfen befindet oder nicht oder wie sich die Bevölkerungsmeinung zu einem Thema oder Akteur darstellt. Der Grad der Medialisierung des Handelns politischer Akteure schwankt dabei offenbar von immens bis kaum vorhanden. Diese Dynamiken und Randbedingungen medialer Einflüsse auf die Politik müssen künftig sehr viel stärker beachtet werden. Sie müssen beschrieben und erklärt werden. Nur so kann auch ihr langfristiger Wandel befriedigend erklärt werden.

Aus empirischer Sicht verlangt ein dynamisches Verständnis von Medialisierung natürlich vor allem Längsschnittstudien. Sie liegen bislang nur sehr vereinzelt vor, was auch daran liegt, dass die methodischen Möglichkeiten retrospektiver Untersuchungen naturgemäß beschränkt sind. Die vorliegenden Längsschnittstudien konzentrieren sich deshalb in erstere Linie auf Medieninhalte oder schriftliche Quellen, die z. B. Aussagen über organisatorische Veränderungen von Parteien oder die Entwicklung parlamentari-

44 Z.B Schrott, Dimensions: Catch-All Label OR Technical Term, aaO. (FN 21), S. 41-61.

45 Z.B. Donges, Medialisierung politischer Organisationen. Parteien in der Mediengesellschaft, aaO. (FN 32).

46 Z.B Frank Marcinkowski, / Adrian Steiner, »Was heißt `Medialisierung‘? Autonomiebeschränkung oder Ermöglichung von Politik durch Massenmedien?« in: Klaus Arnold, / Christoph Classen, / Egard Lersch, / Susanne Kinnebrock, / Hans-Ulrich Wagner, (Hg.), Von der Politisierung der Medien zur Medialisierung des Politischen? Zum Verbältnis von Medien und Politik im 20. Jabrbundert, Leipzig 2010.

47 Z.B. Hartmut Esser, Soziologie. Spezielle Grundlagen. Band 1: Situationslogik und Handeln, Frankfurt/New York 1999. 
scher Aktivitäten erlauben. ${ }^{48}$ In einigen Untersuchungen werden politische Akteure auch um retrospektive Einschätzungen von Veränderungen und Urteilen über die Ursachen der Medialisierung gebeten. ${ }^{49}$ Das bedeutet aber nicht, dass Querschnittsstudien, die den Status quo von Medieneinflüssen auf die Politik untersuchen, für die Medialisierungsforschung unnütz seien. Denn auch eine Zusammenschau entsprechender Befunde aus verschiedenen Zeiten kann über den Stand und die Entwicklung der Medialisierung Auskunft geben. Für die künftige Forschung bedeutet dies nicht nur, dass bisherige Studien zu Medieneinflüssen auf die Politik systematisch aufgearbeitet werden und neue Quellen und Indikatoren erschlossen werden müssen. Es bedeutet vor allem, dass eine Medialisierungsforschung, die sozialen Wandel in den Interaktionen von Medien und Politik über längere Zeit nachzeichnen will, heute beginnen und über einen längeren Zeitraum fortgeführt werden muss.

\section{Zusammenfassung}

Über eine mögliche Medialisierung der Politik wird schon geraume Zeit diskutiert. Dennoch werden immer wieder erhebliche theoretische und empirische Defizite der Forschung bemängelt. Wesentliche Ursachen dafür sind begriffliche Unschärfen und konzeptuelle Mängel. Diese abzubauen, das Konzept der Medialisierung schärfer zu konturieren und für die künftige Forschung besser greifbar zu machen, ist das Ziel des vorliegenden Beitrags. Ausgangspunkt ist ein Verständnis von Medialisierung als dynamischer Prozess sozialen Wandels auf Seiten von politischen Akteuren, Bürgern und/oder Medien. Im Anschluss daran werden die möglichen Ursachen, Indikatoren und Folgen der Medialisierung des Handelns politischer Akteure in den Blick genommen und diskutiert. Dabei wird u.a. dafür plädiert, in stärkerem Maße außerhalb der Medien verortete Ursachen der Medialisierung zu berücksichtigen, die Wahrnehmungen der Akteure in den Blick zu nehmen, sich stärker mit Medieneinflüssen auf die Substanz politischer Entscheidungen zu beschäftigen und die Frage der Medialisierung im Rahmen akteursorientierter, struktur-individualistischer Theorien zu bearbeiten.

\section{Summary}

The mediatisation of politics has been discussed for a fairly long time. However, there still is a lot of criticism pointing to both theoretical and empirical deficits of research.

48 Z.B. Hans Mathias Kepplinger, »Kleine Anfragen. Funktionale Analyse einer parlamentarischen Praxis « in: Werner J. Patzelt, / Uwe Kranenpohl, / Martin Sebaldt, / Heinrich Oberreuter, (Hg.), Res publica semper reformanda. Wissenschaft und politische Bildung im Dienste des Gemeinwobls; Festschrift für Heinrich Oberreuter zum 65. Geburtstag, Wiesbaden 2007, S. 304-319; und Vowe, Mediatisierung der Politik? Ein theoretischer Ansatz auf dem Prüfstand, aaO. (FN 13), S. 433-436.

49 Z.B. vgl. Pontzen, Nur Bild, BamS und Glotze? Medialisierung der Politik aus Sicht der Akteure, aaO. (FN 5); Donges, Medialisierung politischer Organisationen. Parteien in der Mediengesellschaft, aaO. (FN 32). 
Those deficits can at least partly be explained by the terminological fuzziness and conceptual problems related to the mediatisation thesis. The purpose of this paper is to address those shortcomings. It tries to make the concept of mediatisation more concrete and easier to handle in future research. The starting point of the article, then, is a definition of mediatisation as a dynamic process of social change among political actors, citizens and/or the media. In the following, possible causes, indicators and effects of the mediatisation of political actors' actions are focussed and discussed. Among other things the authors argue that research should look more into possible causes of mediatisation outside the media, to take the role of individual perceptions more seriously, to focus more on media effects on the substance of political decisions and to discuss the mediatisation of political actors in the context of actor-centered, structural-individualistic approaches.

Carsten Reinemann, Mediatisation without end? Reflecting the debate about media influences on politics

\section{Politik und Religion}

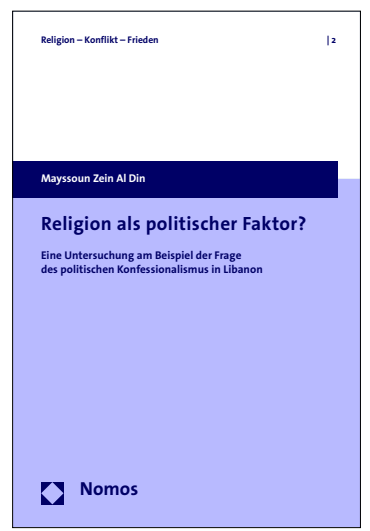

Religion als politischer Faktor?

Eine Untersuchung am Beispiel der Frage des

politischen Konfessionalismus

in Libanon

Von Mayssoun Zein Al Din

2010, 290 S., brosch., 44,- $€$,

ISBN 978-3-8329-5360-7

(Religion - Konflikt - Frieden, Bd. 2)

Im Zentrum der Studie steht die umstrittene Machtverteilungsproblematik des politischen Systems Libanons mit der Erfahrung von Krieg und permanenter Instabilität. Die Autorin schlägt einen ganzheitlichen Erklärungsansatz des libanesischen Konfessionalismus vor und leistet einen Beitrag zur Erforschung der Funktion von Religion in multikonfessionellen Gesellschaften.

Bitte bestellen Sie im Buchhandel oder versandkostenfrei unter $\boldsymbol{\sim}$ www.nomos-shop.de

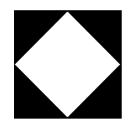

Nomos 